\title{
Hepatic Epithelioid Hemangioendothelioma Diagnosed by Gd-EOB-DTPA-Enhanced MR
}

\author{
Peng $\mathrm{Xu}{ }^{1} \cdot$ Jun $\mathrm{Li}^{1}$ (D) \\ Received: 15 December 2020 / Accepted: 20 December 2020 / Published online: 15 January 2021 \\ (C) 2021 The Society for Surgery of the Alimentary Tract
}

\begin{abstract}
A 39-year-old man was referred to us for evaluation of asymptomatic masses in the liver that had been detected on ultrasonography performed during a physical screening. He had no history of hepatitis and was otherwise well. The level of glutamyl transpeptidase was elevated $(61.1 \mu / \mathrm{L}$; normal level, 3-50 $\mu / \mathrm{L}$ ). Tumor markers were normal, including alpha fetoprotein, carcinoembryonic antigen, and cancer antigen 19-9. No tumor was found through gastroscopy and colonoscopy. GdEOB-DTPA-enhanced MR (Fig. 1) demonstrated multiple peripheral location of lesions with decreases in Gd-EOB-DTPA uptake. The lollipop sign, the target sign, and the capsular retraction were also revealed. The MR findings were highly suggestive of hepatic epithelioid hemangioendothelioma (HEH).
\end{abstract}

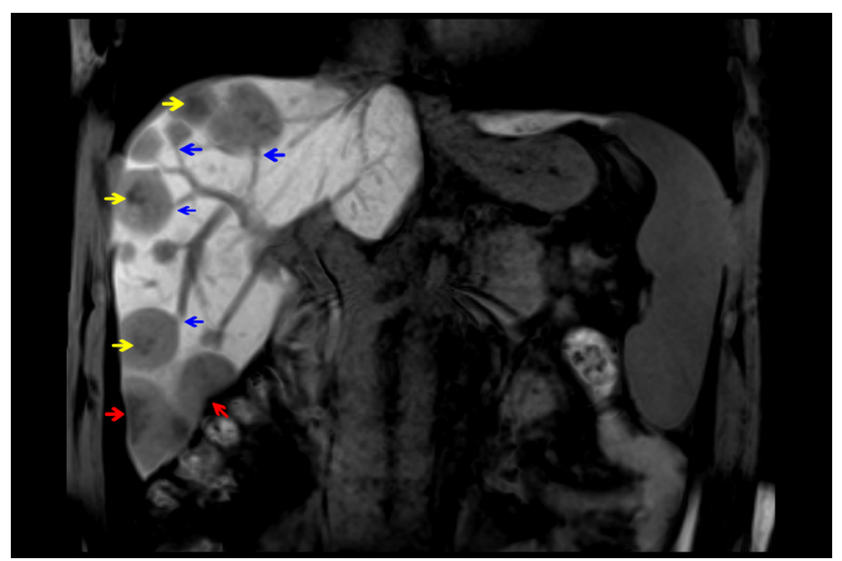

Fig. 1 Hepatobiliary phase of Gd-EOB-DTPA-enhanced MR demonstrated multiple low uptake lesions with hepatic or portal veins entering and terminating in the periphery of the lesion (blue arrows), forming the lollipop sign. Most lesions showed more sclerotic fibrous center (yellow arrows), forming the target sign. The capsular retraction (red arrows) and the coalescence of multiple lesions were also revealed

Jun Li

bzmceducn@sina.com

1 Department of Radiology, Yantai Affiliated Hospital of Binzhou Medical University, Yantai 264100, Shandong, China
Pathological examination of tissue obtained by means of percutaneous biopsy confirmed the diagnosis, with the following immunohistochemistry results: CD31 (+), CD34 (+), D2-40 (+), ERG (+), FLI-1 (+), SMA (+), EMA (-), and Ki-67 (3\%+). Hepatic epithelioid hemangioendotheliomas are rare liver tumors of vascular origin with a low incidence. Peripheral location of the nodules, the lollipop sign, a target-like appearance, capsular retraction, and coalescence of multiple lesions are characteristic radiological findings of $\mathrm{HEH}^{1}$ Repeat Gd-EOB-DTPAenhanced MR 3 months later showed no evident change of the lesions. To our knowledge, this is the first description of GdEOB-DTPA-enhanced MR for diagnosing hepatic epithelioid hemangioendothelioma.

\section{Compliance with Ethical Standards}

Conflict of Interest The authors declare that they have no conflict of interest.

\section{Reference}

1. Mamone G, Miraglia R. The "Target sign" and the "Lollipop sign" in hepatic epithelioid hemangioendothelioma. Abdom Radiol (NY) 2019;44(4):1617-1620. https://doi.org/10.1007/s00261-018-1820-9.

Publisher's Note Springer Nature remains neutral with regard to jurisdictional claims in published maps and institutional affiliations. 